\title{
Antropología
}

\section{Descubriendo caminos: la relación enfermera-familia en comunidades rurales en la Amazonía peruana}

\section{Discovering roads: the relationship nurse - family in rural commu- nities of the Peruvian Amazon}

\section{Descobrindo caminhos: o relacionamento enfermeira-família em comunidades rurais da Amazônia peruana}

${ }^{1}$ Chávez Alvarez, Rocio Elizabeth, ${ }^{2}$ Angelo, Margareth, ${ }^{3}$ Hoga, Luiza Akiko Komura

${ }^{1}$ Doctoranda en Cuidados de la Salud, Programa de Post-Grado en Enfermería. Universidad de Sao Paulo (USP). Brasil. ${ }^{2}$ Ph.D. Profesor Principal. Departamento de Enfermería Materno Infantil y Psiquiátrica. Escuela de Enfermería. USP. Brasil. ${ }^{2}$ Ph.D. Profesor Libre-Docente. Departamento de Enfermería Materno Infantil y Psiquiátrica. Escuela de Enfermería. USP. Brasil.

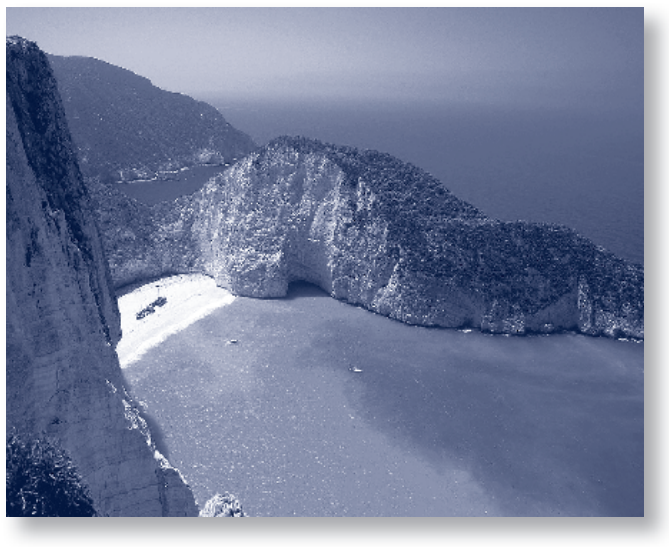

Discovering roads: the relationship nurse family in rural communities of the Peruvian Amazon

\section{ABSTRACT}

From the experience lived by one of the authors as a community nurse in Peru, this reflection had as aim to describe and to understand the meaning and sense found in the relationship between nurse and families in rural communities. The Interpretative Interacionism permitted us the analysis of the experience, where "Discovering roads" was the main component of the experience. The valorization of nurse-family relationship, in a comprehensive and humanized way, allowed to carry out the basic actions with the families. The steps followed were: Inserting in their world; Knowing their reality, Perceiving their vulnerability; Understanding their concerns; Perceiving their ways of acting; Sharing the activities. In the description of the meanings attributed to the experience, contextual aspects, actions and intentions of the nurse and the feelings emerged during the implementation of the activities with the families, were explored. This work enabled us to understand the meaning of the experience, rescuing the importance of nurse-family relationship, making appropriate use of communication, observation and interaction, as well as, the use of strategies to achieve walking toward cultural maturity and humanized care, together and, in order to solve some crucial problems and needs that emerge in this context.

Key-words: Professional-family relations. Family health. Family nursing. Community health nursing. Rural health. 
Descobrindo caminhos: o relacionamento enfermeira-família em comunidades rurais da Amazônia peruana

\section{RESUMO}

A partir da experiência vivenciada por uma das autoras enquanto enfermeira comunitária no Peru. Esta reflexão teve como objetivo descrever e compreender o significado e o sentido encontrados no relacionamento enfermeira-família em comunidades rurais. $\mathrm{O}$ Interacionismo Interpretativo permitiu a análise da vivencia, onde "Descobrindo caminhos" constituiu-se o componente principal da experiência. A valorização do relacionamento enfermeira-família de forma integral e com cuidado humanizado permitiu o cumprimento das ações básicas previstas no trabalho com as famílias. Os caminhos percorridos foram: Inserindo-se no mundo, Conhecendo sua realidade, Percebendo sua vulnerabilidade, Compreendendo suas preocupações, Percebendo sua forma de agir e, Compartilhando as atividades. Ao descrever os significados atribuídos à experiência foram explorados aspectos contextuais, ações e intenções da enfermeira e os sentidos encontrados durante as atividades desenvolvidas com as famílias. Compreender o significado da experiência permitiu resgatar a importância do relacionamento enfermeirafamília, com o uso adequado da comunicação, observação e interação e utilizando estratégias para uma caminhada conjunta em direção ao amadurecimento cultural e o cuidado humanizado, permitindo a resolução de alguns problemas e necessidades prioritários nesse contexto.

Palavras-chave: Relações profissional-família. Saúde da família. Enfermagem familiar. Enfermagem em saúde comunitária. Saúde da população rural.

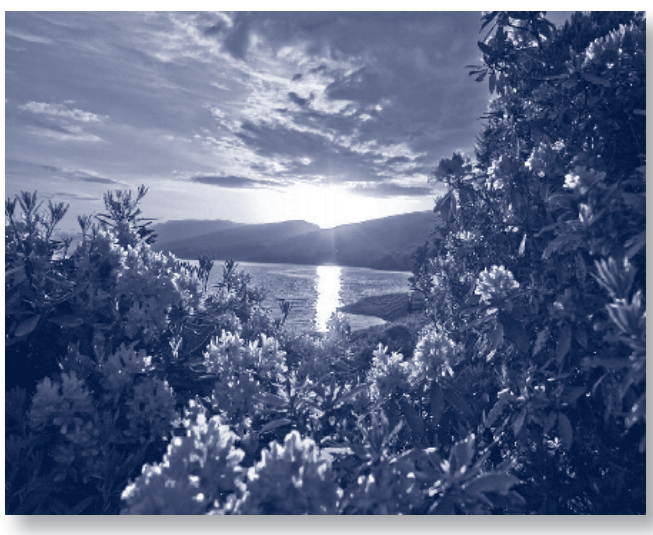

\section{RESUMEN}

Partiendo de la experiencia vivida por una de las autoras como enfermera comunitaria en Perú, esta reflexión tuvo como objetivo describir y comprender el significado y sentido encontrados en la relación enfermera-familia en comunidades rurales. El Interaccionismo Interpretativo permitió el análisis de la vivencia, donde "Descubriendo caminos" se constituyó el componente principal de la experiencia. La valoración de la relación enfermera-familia de forma integral y con cuidado humanizado dio paso al cumplimiento de las acciones básicas previstas en el trabajo con las familias. Las etapas recorridas fueron: Insertándose en su mundo, Conociendo su realidad, Percibiendo su vulnerabilidad, Comprendiendo sus preocupaciones, Percibiendo su forma de actuar y, Compartiendo las actividades. Al describir los significados atribuidos a la experiencia fueron explorados aspectos contextuales, acciones e intenciones de la enfermera y los sentidos encontrados durante las actividades desarrolladas con las familias. Al comprender el significado de la experiencia se rescató la importancia de la relación enfermera-familia, haciendo uso adecuado de la comunicación, observación e interacción y utilizando estrategias para un caminar juntos hacia la madurez cultural y el cuidado humanizado, que permi- 
tieron la resolución de algunos problemas y necesidades prioritarios en dicho contexto.

Palabras-clave: Relaciones profesionalfamilia. Salud de la familia. Enfermería de la familia. Enfermería en salud comunitaria. Salud rural.

\section{INTRODUCCIÓN}

\section{Caracterización y contexto de las comunida-} des rurales

El trabajo como enfermera durante varios años con familias en comunidades rurales del Perú, posibilitó la vivencia de muchas experiencias en la relación enfermera-familia. La descripción y análisis de las interacciones desarrolladas con estas familias, el trabajo cotidiano con esos grupos comunitarios, y por tratarse de una experiencia en particular, aun no está descrita en la literatura científica.

La descripción sistematizada de esta experiencia propone contribuir con el conocimiento previo y las adecuadas relaciones interpersonales de los profesionales de salud que desarrollan trabajo asistencial y de investigación junto a estas comunidades rurales. Lo que permitiría un mejor desarrollo de sus actividades y una relación con los nativos de una determinada cultura de forma empática, humanizada y con la satisfacción de todos los participantes.

A partir de esta experiencia se describen y analizan las interacciones desarrolladas con familias de comunidades rurales, entre ellos pueblos indígenas de la Amazonia Peruana.

El panorama del Sistema de Salud Público Nacional está aún en proceso de innovación, implementando actividades de promoción de la salud, prevención de enfermedades y educación en salud en las comunidades rurales. Son abordados generalmente los grupos de mayor riesgo como el binomio madre-niño, pacientes en extrema pobreza y marginalidad, por medio de los programas básicos de salud que en teoría, llegan hasta las comunidades y aldeas más distantes, necesitadas y apartadas del país (Perú, 1999, 2003).

El equipo de salud comunitario está conformado por médico, enfermera, obstetriz, odontólogo y otros, sin embargo, apenas existe un único profesional o técnico de salud en la mayoría de localidades más apartadas.

La unidad básica de salud es llamada "puesto de salud o centro de salud" $\mathrm{y}$, ni siempre está totalmente equipada, por lo que en algunas ocasiones recibe apoyo logístico de instituciones no-gubernamentales o filantrópicas que trabajan en las zonas aledañas, para que recursos humanos recién egresados de la Academia actúen en el Servicio Rural y Urbano Marginal en Salud (SERUMS), regido por el Ministerio de Salud (PERU, 1999).

La situación de salud y educación de la población en las áreas rurales, es aún preocupante, careciendo en su mayoría de servicios básicos de agua y alcantarillado (OMS, 1993), no obstante, cuentan con algunos recursos locales como la escuela inicial y/o primaria, botiquín de salud, club de madres o comedor popular, implementados con recursos mínimos diversos, mas a veces, sin las condiciones básicas necesarias para su adecuado funcionamiento.

El índice de enfermedades prevenibles en las comunidades rurales es aún elevado, siendo las enfermedades diarreicas, infecciones respiratorias y dermatológicas, parasitosis y problemas gineco-obstétricos, entre otros, los más emergentes (Reátegui, 2005); así también el saneamiento básico es precario que, ligado al bajo nivel educativo refleja en los niños problemas de desnutrición y/o enfermedades infecto-contagiosas (Perú, 2003), resultado también, de las desigualdades de esas poblaciones 
en relación con otros grupos sociales, lo que se expresa en altas tasas de morbi-mortalidad materno infantil (OMS, 1993, Reátegui, 2005).

Por otro lado, la enfermería comunitaria en el Perú comenzó a desarrollarse lentamente después de la década de los 50 s, con programas de salud básicos, actividades de detección pre$\mathrm{coz}$ y seguimiento de casos en la comunidad y énfasis en la atención preventivo-promocional de la familia y grupos de riesgo, así como la educación e inmunizaciones, con tendencia a la integralidad de la familia, del escolar, del trabajador y del medio ambiente, aunque el punto de partida haya sido el individuo enfermo como objeto que tiene el daño (Pecho, 2004).

Por tanto, partiendo de esta visión general y del contexto de trabajo con familias, el presente estudio se planteó como objetivo describir y comprender el significado y el sentido encontrados en la relación enfermera-familia en comunidades rurales. A fin de concretizar y encaminar las actividades de enfermería a los grupos prioritarios, intentando discutir $y$ resolver los problemas y/o necesidades comunitarias relevantes de forma conjunta y con un cuidado humanizado.

\section{La relación enfermera-familia en la comuni- dad}

Este trabajo de reflexión de la experiencia con familias de comunidades rurales, trae a la luz algunos elementos que consideramos los caminos recorridos como producto de la interacción, colocando nuestra comprensión de los significados de la relación enfermerafamilia en este contexto determinado.

Para la obtención de los elementos analizados, se hace uso de la narración autobiográfica, relacionando los eventos significativos de la experiencia vivida y colocándolos en el contexto, resultado del análisis (Bergamasco, 1999).

Por medio del Interaccionismo Interpretativo (Bergamasco, 1999), cuyo foco reside en las experiencias vividas que alteran o forman el significado de sí mismas, teniendo como presupuesto básico la importancia de la interpretación y de la comprensión como clave que forma la vida social, se dio paso al tema principal "Descubriendo Caminos", que presentamos a seguir, describiendo los elementos que consideramos importantes en el proceso de trabajo con las familias en la comunidad.

\section{Descubriendo caminos}

Este tema se configura como el componente principal de la relación, siendo que, por medio de la interacción, comunicación y observación durante el tiempo de convivencia con las comunidades rurales se consiguió entrar en el mundo de las familias, comprendiendo su vida diaria y sus caminos recorridos en el cuidado de la salud-enfermedad, el trabajo y la vida comunitaria (Pettengill, 1998).

De tal forma, se desvelan algunos elementos utilizados en el proceso de trabajo de este estudio que se constituyeron en etapas fundamentales del camino recorrido con las familias de las comunidades rurales, con miras al mejor desempeño en la práctica de enfermería.

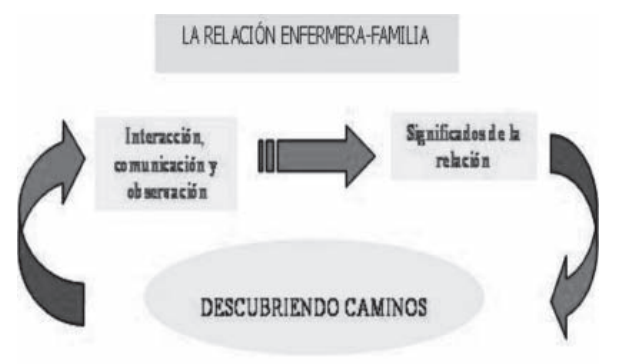

FIGURA 1. La relación enfermera-familia: Descubriendo caminos. Sao Paulo, 2009. (anexo 1) 


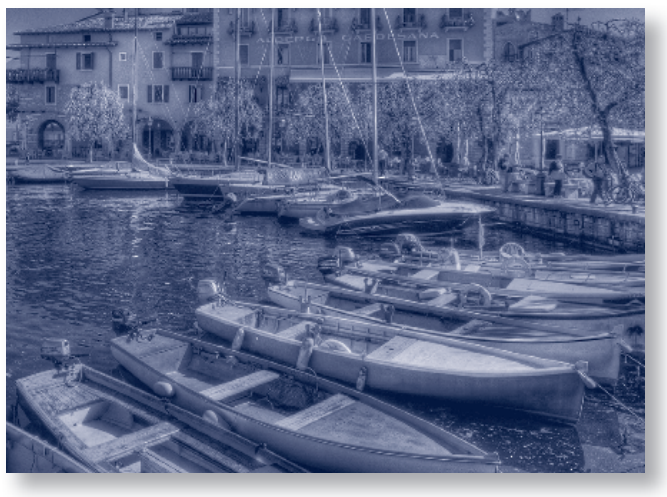

Componentes de la relación enfermera-familia en la comunidad

\section{1- Insertándose en su mundo}

Fue fundamental como enfermera integrarse a la comunidad de forma comprometida. Al trabajar con las familias se percibió una relación cada vez más envolvente, llegando a valorarlos como personas e intentando colocarse en el lugar de ellas, de esta manera se percibía el crecimiento de la comunidad día a día y se ampliaba mi propia visión de mundo.

"Ser presentada a los miembros de la comunidad, sentirme al comienzo un poco temerosa por la expectativa, fue el punto inicial de la relación; mas poco a poco los temores se alejaban al percibir la calurosa acogida y las expectativas que tenían, tanto las madres de familia, como los representantes de la comunidad" (Amazonas, 1998-1999).

La experiencia de trabajo con familias y comunidades siempre tiene un comienzo y aunque el futuro sea incierto, estar con la mente y la disposición abiertas ayuda a iniciar los nexos de una relación enfermera-familia con mutuo crecimiento. Para ello, es importante reconocer a la familia como una persona con sus características y necesidades particulares (Wernet, Angelo, 2003). Así, la atención de salud se vuelve más humanizada, estableciendo relaciones adecuadas entre los usuarios y los profesionales de salud (Hoga, 2004).

\section{2- Conociendo su realidad}

Una vez integrada a la comunidad y conociendo las reales condiciones en que vivían las familias, es que se consigue ir más allá del cuidado, con medidas que puedan concretarse $y$ promuevan calidad de vida a partir del cuidado de enfermería y la participación de las familias.

"Las familias manifestaron sus dificultades para obtener agua potable y desagüe, de los cuales carecían. Por lo que se establecieron acuerdos pacientes y constantes con las empresas aledañas para que contribuyan, así como, las familias se organizaban para el trabajo comunitario $y$ educativo en la comunidad, resultando en algunas soluciones razonables de acuerdo a su realidad" (Huánuco y Ucayali, 2005).

Conocer las condiciones socio-económicas, necesidades y carencias, creencias y valores culturales y otros aspectos de una población determinada, contribuyen con el compromiso y mejor desempeño de los profesionales de salud con sus clientes, para la búsqueda de resultados coherentes y concretos con la realidad de vida de las personas bajo su cuidado (Hoga, 2004), así como, contar con el soporte de las redes sociales del entorno.

\section{3- Percibiendo su vulnerabilidad}

Momentos de fragilidad al enfrentar algunas situaciones críticas de su cotidiano, eran observadas en las familias, donde era evidente el papel de la mujer dentro de la comunidad, al organizarse para resolver ciertos problemas.

Sentimientos encontrados surgían al percibir su vulnerabilidad, sentimientos que trataban de controlarse y transformarse positivamente para motivar a las familias a continuar su camino y valorizarlos como un grupo que progresaba.

"Cierta vez, una adolescente con su bebé en los brazos que lloraba desesperadamente, busca- 


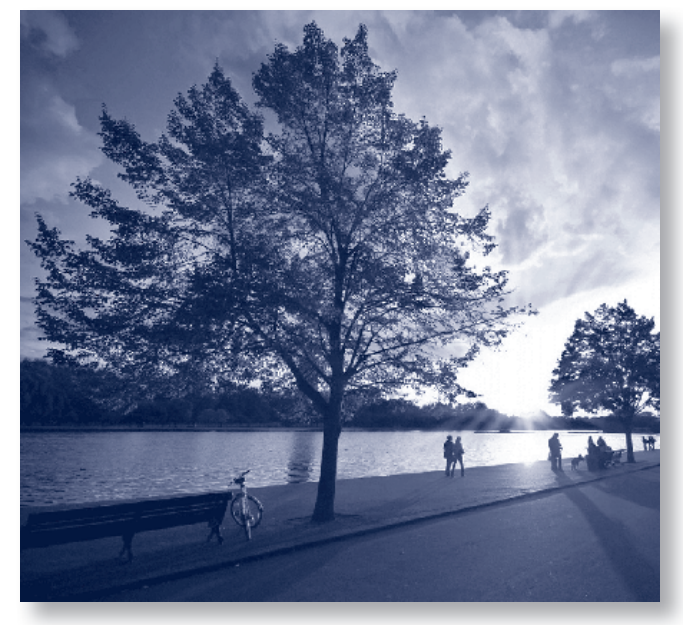

ba ayuda porque no podía calmarlo, después de recibirla, acogerla y brindarle orientación, ella consiguió amamantar a su recién nacido, luego nos sentamos a conversar, manifestando que vivía sola, no tenía esposo y no había planificado ser madre..., más tarde, fue integrada y acogida en el club de madres de la comunidad" (Huancavelica, 2004).

Aspectos de vulnerabilidad de las personas se exponen al encuentro con situaciones inesperadas, por lo que un medio útil para resolverlos se deriva de la reflexión conjunta sobre la situación, lo que lleva a despejar el problema y canalizar mejor las vías o alternativas de apoyo que la comunidad y el profesional de salud puedan brindar.

Muchas veces no hacen falta técnicas o mecanismos complejos, simplemente brindar cariño, ayuda y realizar acciones que expresen cuidado en dichas situaciones (Chávez, 2002). Así también, el auto-conocimiento del profesional de salud, el conocerse a si mismo, posibilita ser consciente de las propias limitaciones y fragilidades, lo que permite descubrir y utilizar de mejor forma las propias potencialidades (Hoga, 2004) para un cuidado humanizado de las personas más susceptibles que pasan por el evento o situación inesperada.

\section{4- Comprendiendo sus preocupaciones}

Al reflexionar sobre la complejidad de las interacciones familiares, intentaba colocarme en el lugar de las familias, tratando de comprender sus miedos, angustias, alegrías y tristezas de forma real y verdadera; saber por ellas mismas que situaciones cotidianas enfrentaban y que inseguridades tenían. Así, por medio de la interacción, las familias transmitían sus inquietudes y preocupaciones, organizándose luego para actuar en grupo y resolverlas.

"Las familias querían saber cómo podían disminuir los problemas de diarreas y parasitosis en los niños de su comunidad, fue así que comprendida esa necesidad, se unieron esfuerzos y efectuaron actividades educativas y de intervención en salud, con las empresas aledañas, los comuneros y las escuelas de la comunidad" (Amazonas, 1998; Loreto, 1999; Ucayali, 2005).

Las familias estaban preocupadas generalmente por la salud de sus hijos, en su mayoría afectados por enfermedades comunes y se sentían a veces sin el apoyo de los profesionales de salud del Puesto o Centro de Salud, por lo que atribuían sus dificultades a fallas externas en el sistema local de salud pública, como la falta de personal o escasez de materiales (OMS, 1993). Delante de esas situaciones, buscaban solucionar sus necesidades con sus recursos locales, pidiendo ayuda al médico curandero, la partera $y / o$ el promotor de salud comunitario (Chávez et.al., 2007).

"La partera y el médico curandero eran buscados muchas veces por las madres, para atender problemas de salud de sus hijos o de algún miembro de sus familias, ellos trataban casos de sustos, mal de ojo, diarreas, fiebres no especificas, partos, etc; con hierbas, plantas medicinales, rezos, sesiones de shamanismo y otros; luego los pacientes acudían al Puesto de salud más cercano, si era necesario" (Ucayali, 2006, 2007). 


\section{5- Percibiendo su forma de actuar}

En reuniones organizadas, con frecuencia las familias tomaban decisiones internas a favor de su comunidad o solicitaban apoyo externo para ello. Siendo observado con detenimiento sus actitudes en situaciones de saludenfermedad, familia y trabajo. Esto permitió una comprensión de su dinámica familiar, sus reacciones y su compromiso como familias en el contexto en que vivían. Esa percepción estimulaba el desarrollo de estrategias e instrumentos que unidos al saber científico, impulsaban toda la capacidad humana y profesional para una práctica de enfermería congruente y competente con las necesidades (Purnell, 2002) de las familias en la comunidad.

"Las familias preocupadas con la educación de sus hijos, transmitieron por medio de las escuelas sus necesidades de informarse mas sobre la salud de sus niños, por lo que se desarrollaban actividades de educación básica en salud en las escuelas de inicial y primaria, acciones de promoción de la salud y prevención de enfermedades comunes y emergentes, acordes con sus reales y potenciales necesidades". (Amazonas, 1998, Ucayali, 2007)

Una vez más, como grupo organizado, actuaban frente a sus problemas más prioritarios, movilizándose todos lo que hacían parte o acompañaban el cotidiano de la comunidad.

"Una joven nativa de 19 años, primigesta, dio a luz con dificultad antes de los 7 meses de embarazo, el bebé nació con bajo peso, semi-frío $y$ leve dificultad respiratoria. Ambos se recuperaron rápidamente, madre y recién nacido. Se observaba gran movimiento dentro de la casa, los parientes y vecinos traían cosas cuando era solicitado (agua caliente, trapos limpio, tijeras, algodón, cajas de cartón, yodo, plantas, etc), las mujeres cocinaban hierbas y preparados que daban a la parturiente cuando era indicado, la partera estaba junto a la madre todo el tiempo, así como la abuela materna, el abuelo curandero rezaba y proclamaba cánticos para atraer a los buenos espíritus. La técnica de enfermería, el médico del puesto de salud vecino y nosotras (enfermeras visitantes) también fuimos autorizados para apoyar en algunos cuidados posteriores de la madre y el recién nacido". (Ucayali, 2006)

Al comprobar como la familia y comunidad se organizaba y actuaba frente a sus necesidades y conforme a sus valores creencias y prácticas culturales, los que participamos de esos momentos de interacción nos sentimos gratificados, principalmente los profesionales de salud que, de simples espectadores pasamos a una mejor posición para actuar brindando algunos cuidados culturalmente congruentes y que promuevan oportunidades de mantenimiento de la salud y bienestar en los procesos de salud-enfermedad (Purnell, 2002).

\section{6- Compartiendo las actividades}

Una vez recorridas las etapas anteriores, la seguridad y confianza para trabajar con las familias de la comunidad se consolidaban, considerando siempre sus propias decisiones y haciendo un intento por comprender y respetar sus creencias, costumbres y prácticas, para ello era necesario envolverse en el cuidado como "colaboradora" y no como simple "realizadora de tareas" de enfermería.

Como enfermera, era importante estar sensibilizada y compartir no sólo el cuidado sino también sus experiencias como familias, para poder incorporar un cuidado humanizado $y$ acorde con sus necesidades, con compromiso profesional y empatía en las relaciones con las familias de la comunidad.

"Se planificaron e implementaron acciones de enfermería, posibles de soluciones conjuntas 
con la participación de las familias; así, algunos problemas de diarreas fueron resueltos desde la comunidad, ya que el sistema de salud público tenía poca efectividad y mucha burocracia. Las familias estaban dispuestas a aprender y conocer mejor sobre cómo prevenir enfermedades en sus niños; así, con reuniones comunitarias, en las escuelas y en los hogares, se integró un aprendizaje simple, ameno, dinámico y con resultados favorables para los niños en las escuelas y en la comunidad". (Ucayali, 2006-2007).

Pretender mejorar la situación de salud en la comunidad podía ser posible, si caminábamos juntos uniendo la práctica, el conocimiento y la experiencia de los que hacíamos parte de esa convivencia, de forma que al descubrir algunos caminos alternativos para recorrer juntos dejábamos de lado el trabajo rutinario y la convivencia pasiva y monótona.

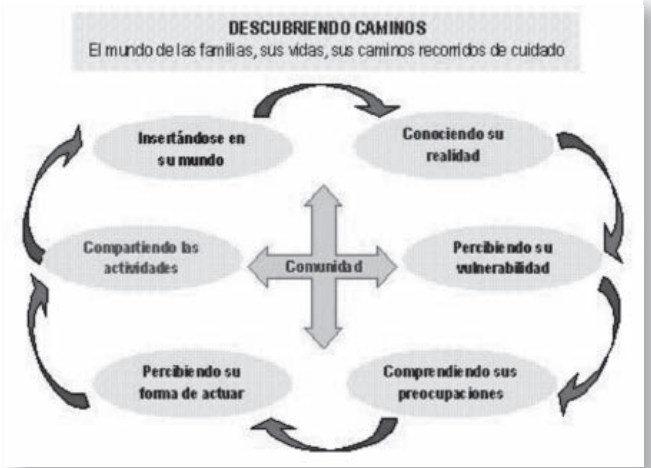

FIGURA 2. Descubriendo caminos - El mundo de las familias en la comunidad. Sao Paulo, 2009. (anexo 1)

\section{Concretizando el trabajo con las comunida- des rurales}

En el proceso de trabajo con las comunidades rurales fueron considerados los aspectos culturales, las creencias, valores y conocimientos generados a partir de las propias experiencias de vida de las familias (Purnell, 2002). Haciendo posible una relación profesionalfamilia favorable y llegando a visualizar a la comunidad en su universalidad y especificidad (Leininger, 2002).

Desempeñarse como profesional de enfermería no hubiera sido posible sin la acogida, abertura y aceptación de cada una de las comunidades por donde se transitó. De tal manera que, las acciones conjuntas fueron consecuencia del proceso de descubrir caminos a través de la convivencia mutua, y después de muchas conversaciones, discusiones, reuniones, con y entre los actores sociales - las familias de las comunidades rurales.

Se dio énfasis a acciones de prevención de enfermedades de la infancia y problemas de salud en las mujeres, además de otras acciones de interés para la comunidad en general, como emergencias y urgencias, tratamiento de enfermedades emergentes, inmunizaciones, manejo del agua, alimentos y desechos, cuidados y visitas domiciliarias, entre otras.

Los niños fueron los principales beneficiados, a medida que se integraba la recreación infantil con el aprendizaje en salud, tanto en las escuelas como en los hogares de las familias.

También se participó de algunas actividades a invitación de las autoridades locales, como fiestas patronales, censos locales, celebraciones sociales intra y extra comunitarias y desfile patriótico, como una forma de integrarse, comprometerse e generar calidad de vida para todos.

\section{Consideraciones finales}

El trabajo efectuado con las comunidades rurales nos permitió conocer y comprender los significados de la experiencia con familias. Por medio de ella fue posible rescatar la importancia de la relación enfermera-familia con el uso adecuado de estrategias de comunicación, observación e interacción, dándonos la oportunidad de caminar juntos hacia una madurez y cuidado mutuo y haciendo posible la reso- 
lución de algunos problemas prioritarios en la comunidad.

Aprender con ellos que las acciones efectivas de cuidado no son fruto de la imposición vertical y occidental de la asistencia en salud, sino del resultado de una convivencia harmónica con respeto por la cultura, valores y creencias dentro del contexto donde viven, (Reátegui, 2005, Chávez et.al., 2007) fue un aprendizaje significativo y trascendental.

Tuvo sentido conocer su realidad, percibir su forma de actuar frente a las situaciones que enfrentaban. Fue interactuando, dando y recibiendo cuidados que pudo visualizarse seres humanos con todas sus posibilidades y capacidades para inter-relacionarse en su cotidiano (Angelo, 1999).

Al introducirse en el mundo de las familias pudo sentirse sus preocupaciones, de tal forma que se alcanzaron algunas intervenciones efectivas en las necesidades de salud prioritarias. De esta manera, fueron descubiertos nuevos caminos en el trabajo con las familias, ya que, el estar sensibilizada e instrumentalizada como enfermera se pudo visualizar alternativas de solución conjunta (Angelo, 1999). Así como, al considerar sus valores, creencias y prácticas culturales, pudo brindarse un cuidado competente y congruente con su realidad que promueva oportunidades de bienestar, promoción de la salud y prevención de riesgos y enfermedades (Purnell, 2002) en las relaciones enfermera-familia en las comunidades donde se transitó.

Fue importante ingresar en el universo cultural de cada comunidad rural, respetando y compartiendo de sus cuidados (Hoga, 2004) y principalmente, resaltando y valorizando el papel que desempeña la mujer-madre (Budó, 1997) dentro de la comunidad, quien ofrecía sus cuidados con integralidad, totalidad y humanización (Chávez et.al., 2007).
Al considerar los procesos sociales y emocionales de las familias, se observó comunidades preocupadas por su salud y calidad de vida, que hacían esfuerzos por ampliar sus conocimientos y mecanismos para enfrentar las diversas situaciones de su cotidiano.

Si bien, la enfermera comunitaria en Perú desarrolla actividades en la comunidad con énfasis en la atención preventivo promocional, (Pecho, 2004) siendo su foco todavía el individuo enfermo, podría decirse que estamos caminando lentamente hacia un cambio por la integralidad de los cuidados con la participación de la comunidad.

Después de la reflexión y compresión de esta vivencia con las familias, consideramos haber recorrido no sólo el camino de la comunidad sino también haber trazado nuestro propio camino, sentando las bases para continuar con nuestro trabajo como enfermeras y para interactuar pertinentemente con personas en cualquier circunstancia de nuestra vida, profesional y personal, sintiendo la plena ciudadanía, creciendo y descubriendo caminos todos los que formamos parte de ese contexto.

\section{REFERENCIAS}

- Angelo, M. (1999) Abrir-se para a família: superando os desafios. Fam Saúde e Desenv. 1(12), 7-14.

- Bergamasco, RB. (1999) O sofrimento de descobrir-se com câncer de mama: como o diagnóstico é experienciado pela mulher [tese de doutorado]. São Paulo: Escola de Enfermagem da Universidade de São Paulo.

- Budó, MLB. (1997) A mulher como cuidadora no contexto de uma comunidade rural de imigração italiana. Texto Contexto Enferm. 6(1), 181-97.

- Chávez Alvarez, RE et al. (2007) Rescatando el autocuidado de la salud durante el embarazo, el parto y al recién nacido: representaciones sociales de mujeres de una comunidad nativa en Perú. Texto Contexto Enferm. 16(4), 680-7.

- Chávez Alvarez, RE. (2002) O significado do cuidado para a criança hospitalizada [dissertação de mestrado]. 
São Paulo: Escola de Enfermagem da Universidade de São Paulo.

- Hoga, LAK. (2004) A dimensão subjetiva do profissional na humanização da assistência à saúde: uma reflexão. Rev. esc. Enferm USP. 38(1), 13-20.

- Leininger, M. (2002) Transcultural nursing: concepts, theories, research and practice. 3th ed. USA: McGraw Hill.

- OMS. (1993) Organización Mundial de la Salud. Documento CD37/20 sobre la iniciativa Salud de los Pueblos Indígenas de las Américas. Washington D.C: OPS. Disponible en: http://www.paho.org/spanish/ad/ths/os/ indig-Resol-V.htm [Consultado el 1.2.2006]

- Pecho IL. (2004) Enfermería comunitaria en Perú: situación actual y perspectivas. Disponible en: http://raec.tripod.com/per.html [Consultado el 20.3.2004]

- Perú. (1999) Ministerio de Salud. Informe final del servicio rural y urbano marginal de salud. Expediente No 3221-99, Resolución Directorial No 243-99/CTAR-PIURA DRSP - OEA - OPER. Piura: MINSA.

- Perú. (2003) Ministerio de Salud. Centro Nacional de Salud Intercultural. Salud de los pueblos indígenas. Lima:
MINSA. Disponible en: http://www.ins.gob.pe/gxpsites/ hgxpp001.aspx2,8,76,O,S,0,MNU;E;10;6;MNS [Consultado el 12.4.2006]

- Pettengill, MAM. (1998) O sentido de cuidar da criança e da família na comunidade: a experiência da aluna de enfermagem [dissertação de mestrado]. São Paulo: Escola de Enfermagem da Universidade de São Paulo.

- Purnell L (2002) A description of the Purnell model for cultural competence. Journal of transcultural nursing. 11(1), 40-46.

- Reátegui Silva, J. (2005) Las políticas de salud de los pueblos indígenas de la amazonía peruana. Asociación Interétnica de Desarrollo de la Selva Peruana- $1^{\text {a }}$ ed. Lima: AIDESEP. Disponible en: http://www.bvsde.paho. org/bvsacd/cd67/PoliticasAmazonia.pdf [Consultado el 01.08.2007]

- Wernet, M; Angelo, M. (2003) Mobilizando-se para a família: dando um novo sentido à família e ao cuidar. Rev. esc. enferm. USP. (Sao Paulo); 37(1), 19-25.

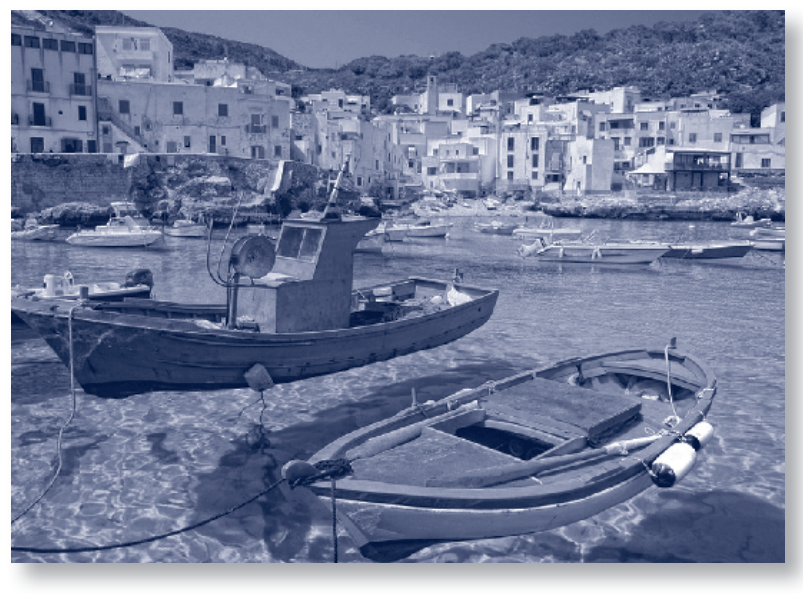

